Qin-Juan Xu*

\title{
Crystal structure of $\mathrm{N}$-(5-(2-(benzyl(1- (4-methoxyphenyl)propan-2-yl)amino)- 1-hydroxyethyl)-2-(benzyloxy)phenyl)formamide, $\mathrm{C}_{33} \mathrm{H}_{36} \mathrm{~N}_{2} \mathrm{O}_{4}$
}

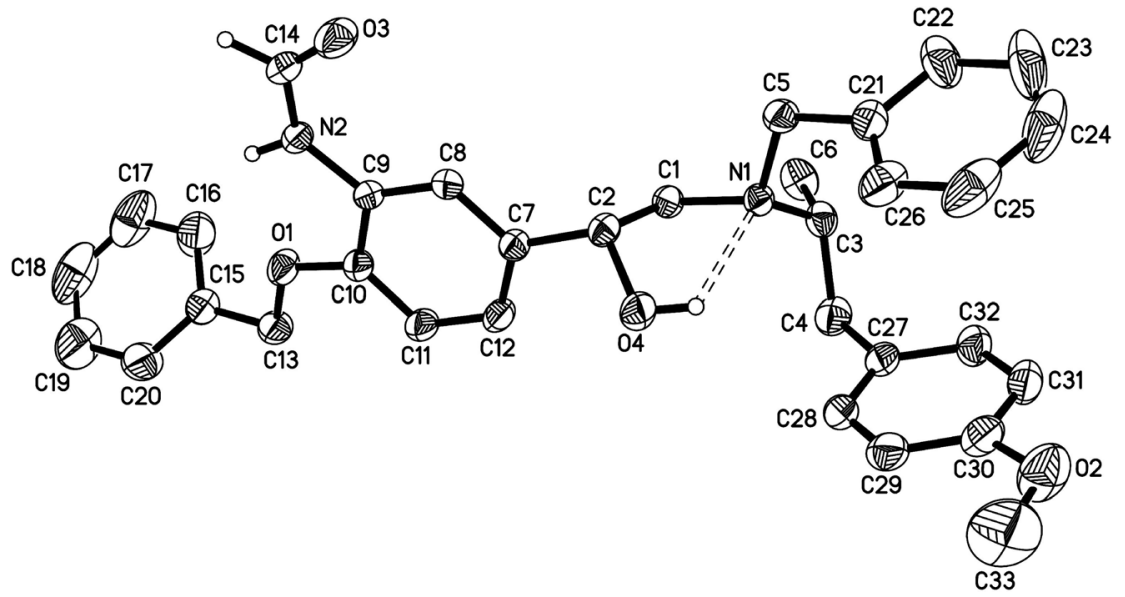

https://doi.org/10.1515/ncrs-2021-0012

Received January 8, 2021; accepted January 28, 2021; published online March 1, 2021

\section{Abstract \\ $\mathrm{C}_{33} \mathrm{H}_{36} \mathrm{~N}_{2} \mathrm{O}_{4}$, orthorhombic, Pbca (no. 61), $a=15.3543(17) \AA, b=14.7958(17) \AA, c=25.496(3) \AA$, $V=5792.1(11) \AA^{3}, Z=8, R_{g t}(F)=0.0490, w R_{r e f}\left(F^{2}\right)=0.1402$, $T=296(2) \mathrm{K}$.}

CCDC no.: 2059329

The molecular structure is shown in the Figure. Table 1 contains crystallographic data and Table 2 contains the list of the atoms including atomic coordinates and displacement parameters.

\section{Source of material}

The title compound was prepared by the reaction of 1-(3-amino-4-(benzyloxy)phenyl)-2-(benzyl(1-(4-methoxy

*Corresponding author: Qin-Juan Xu, Weihai Ocean Vocational College, Rongcheng, Shandong 264300, P. R. China, E-mail: 15662370667@163.com. https://orcid.org/0000-00022910-209X
Table 1: Data collection and handling.

\begin{tabular}{ll}
\hline Crystal: & Colorless sheet \\
Size: & $0.23 \times 0.19 \times 0.05 \mathrm{~mm}$ \\
Wavelength: & Mo $K \alpha$ radiation $(0.71073 \AA)$ \\
$\mu:$ & $0.08 \mathrm{~mm}^{-1}$ \\
Diffractometer, scan mode: & $\varphi$ and $\omega$ \\
$\theta_{\text {max }}$, completeness: & $27.0^{\circ},>99 \%$ \\
$N(h k l)_{\text {measured }}, N(h k l)_{\text {unique }}, R_{\text {int }}:$ & $26962,6305,0.028$ \\
Criterion for $I_{\text {obs }}, N(h k l)_{\mathrm{gt}}:$ & $I_{\text {obs }}>2 \sigma\left(I_{\text {obs }}\right), 4395$ \\
$N(\text { param })_{\text {refined }}:$ & 364 \\
Programs: & Bruker [1], SHELX [2], \\
& Diamond [3] \\
\hline
\end{tabular}

-phenyl)propan-2-yl)amino)ethan-1-ol (R1) with formic acid protected by $\mathrm{N}_{2} .6 .5 \mathrm{~g}$ R1 was dissolved in $30 \mathrm{~mL}$ $\mathrm{ClCH}_{2} \mathrm{CH}_{2} \mathrm{Cl}$. $4.49 \mathrm{~g} \mathrm{EtN}=\mathrm{C}=\mathrm{N}\left(\mathrm{CH}_{2}\right)_{3} \mathrm{NMe}_{2}$ hydrochloric acid salt was dissolved in $25 \mathrm{~mL} \mathrm{ClCH}_{2} \mathrm{CH}_{2} \mathrm{Cl}$ and mixed with the former solution cooled to $10{ }^{\circ} \mathrm{C}$. Then, $1.2 \mathrm{~g}$ anhydrous formic acid was added slowly and reacted for $2 \mathrm{~h}$. After that, the organic phase was washed with $10 \%$ sodium carbonate hydrous solution and $20 \%$ sodium chloride hydrous solution for twice, respectively. The organic phase was concentrated at $40-50{ }^{\circ} \mathrm{C}$ to give rise to dark-red stickiness substance (yield 68\%). This method was referenced to a patent [4]. The colorless sheet-shaped crystals suitable for $\mathrm{X}$-ray diffraction of the title compound were obtained by slow evaporation of a $\mathrm{MeOH}$ solution. 
Table 2: Fractional atomic coordinates and isotropic or equivalent isotropic displacement parameters $\left(\AA^{2}\right)$.

\begin{tabular}{|c|c|c|c|c|}
\hline Atom & $x$ & $y$ & $z$ & $U_{\text {iso }} * / U_{\text {eq }}$ \\
\hline N1 & $0.46246(8)$ & $0.12243(8)$ & $0.83595(5)$ & $0.0406(3)$ \\
\hline $\mathrm{N} 2$ & $0.09567(9)$ & $0.27618(9)$ & $0.98248(5)$ & $0.0464(3)$ \\
\hline 01 & 0.10064 (8) & $0.14121(8)$ & $1.04744(5)$ & $0.0613(3)$ \\
\hline 02 & $0.87256(10)$ & $0.16534(14)$ & $0.87061(8)$ & $0.1029(6)$ \\
\hline 03 & $0.13010(9)$ & $0.39210(10)$ & $0.92729(6)$ & $0.0751(4)$ \\
\hline 04 & $0.46809(7)$ & $0.18781(9)$ & $0.93622(5)$ & $0.0563(3)$ \\
\hline C1 & $0.38441(9)$ & $0.11814(11)$ & $0.86881(6)$ & $0.0418(3)$ \\
\hline $\mathrm{H} 1 \mathrm{~A}$ & 0.3813 & 0.0595 & 0.8857 & $0.050^{*}$ \\
\hline $\mathrm{H} 1 \mathrm{~B}$ & 0.3331 & 0.1252 & 0.8470 & $0.050^{*}$ \\
\hline $\mathrm{C} 2$ & $0.38579(9)$ & $0.19203(10)$ & $0.91047(6)$ & $0.0426(3)$ \\
\hline $\mathrm{H} 2 \mathrm{~B}$ & 0.3795 & 0.2511 & 0.8935 & $0.051^{*}$ \\
\hline C3 & $0.49060(11)$ & $0.03357(10)$ & $0.81525(6)$ & $0.0479(4)$ \\
\hline $\mathrm{H} 3 \mathrm{~A}$ & 0.5270 & 0.0459 & 0.7845 & $0.058^{*}$ \\
\hline $\mathrm{C} 4$ & $0.54882(12)$ & $-0.01433(12)$ & $0.85524(7)$ & $0.0599(5)$ \\
\hline $\mathrm{H} 4 \mathrm{~B}$ & 0.5191 & -0.0161 & 0.8888 & $0.072^{\star}$ \\
\hline $\mathrm{H} 4 \mathrm{C}$ & 0.5580 & -0.0762 & 0.8440 & $0.072^{\star}$ \\
\hline $\mathrm{C} 5$ & $0.45420(10)$ & $0.19040(11)$ & $0.79426(6)$ & $0.0500(4)$ \\
\hline $\mathrm{H} 5 \mathrm{~A}$ & 0.4279 & 0.2445 & 0.8087 & $0.060^{*}$ \\
\hline $\mathrm{H} 5 \mathrm{~B}$ & 0.4156 & 0.1674 & 0.7673 & $0.060^{*}$ \\
\hline $\mathrm{C} 6$ & $0.41669(13)$ & $-0.02737(13)$ & $0.79671(7)$ & $0.0641(5)$ \\
\hline $\mathrm{H} 6 \mathrm{~A}$ & 0.3817 & 0.0045 & 0.7715 & $0.096^{*}$ \\
\hline H6B & 0.3813 & -0.0443 & 0.8261 & $0.096^{*}$ \\
\hline $\mathrm{H} 6 \mathrm{C}$ & 0.4405 & -0.0807 & 0.7808 & $0.096^{*}$ \\
\hline $\mathrm{C} 7$ & $0.31346(9)$ & $0.17943(10)$ & $0.94947(6)$ & $0.0418(3)$ \\
\hline $\mathrm{C} 8$ & $0.24075(9)$ & $0.23555(10)$ & $0.94766(5)$ & $0.0402(3)$ \\
\hline $\mathrm{H} 8 \mathrm{~A}$ & 0.2390 & 0.2828 & 0.9237 & $0.048^{*}$ \\
\hline C9 & $0.17098(9)$ & $0.22176(10)$ & $0.98123(5)$ & $0.0400(3)$ \\
\hline C10 & $0.17362(10)$ & $0.15047(11)$ & $1.01712(6)$ & 0.0464 (4) \\
\hline C11 & $0.24661(11)$ & $0.09621(12)$ & $1.01994(6)$ & $0.0548(4)$ \\
\hline $\mathrm{H} 11 \mathrm{~A}$ & 0.2493 & 0.0499 & 1.0445 & $0.066^{\star}$ \\
\hline $\mathrm{C} 12$ & $0.31582(10)$ & 0.11084 (11) & $0.98608(6)$ & $0.0511(4)$ \\
\hline $\mathrm{H} 12 \mathrm{~A}$ & 0.3646 & 0.0738 & 0.9881 & $0.061^{*}$ \\
\hline C13 & $0.09412(12)$ & 0.06297 (13) & $1.08000(7)$ & $0.0603(5)$ \\
\hline $\mathrm{H} 13 \mathrm{~A}$ & 0.1346 & 0.0676 & 1.1091 & $0.072^{\star}$ \\
\hline $\mathrm{H} 13 \mathrm{~B}$ & 0.1085 & 0.0093 & 1.0600 & $0.072^{\star}$ \\
\hline C14 & 0.08184 (12) & $0.35480(12)$ & $0.95767(7)$ & $0.0527(4)$ \\
\hline C15 & $0.00311(11)$ & $0.05635(12)$ & $1.10022(7)$ & $0.0547(4)$ \\
\hline C16 & $-0.06654(16)$ & $0.0518(2)$ & $1.06691(10)$ & $0.0966(8)$ \\
\hline $\mathrm{H} 16 \mathrm{~A}$ & -0.0574 & 0.0550 & 1.0309 & $0.116^{*}$ \\
\hline C17 & $-0.15039(18)$ & $0.0426(2)$ & 1.08605 (18) & $0.1218(12)$ \\
\hline $\mathrm{H} 17 \mathrm{~A}$ & -0.1972 & 0.0378 & 1.0630 & $0.146^{\star}$ \\
\hline C18 & $-0.1640(2)$ & $0.0407(2)$ & 1.13904 (19) & $0.1232(13)$ \\
\hline $\mathrm{H} 18 \mathrm{~A}$ & -0.2201 & 0.0344 & 1.1523 & $0.148^{\star}$ \\
\hline C19 & $-0.0951(2)$ & $0.0480(3)$ & $1.17226(14)$ & $0.1300(13)$ \\
\hline $\mathrm{H} 19 \mathrm{~A}$ & -0.1045 & 0.0480 & 1.2083 & $0.156^{*}$ \\
\hline $\mathrm{C} 20$ & $-0.01182(15)$ & $0.05523(18)$ & $1.15302(9)$ & $0.0873(7)$ \\
\hline $\mathrm{H} 20 \mathrm{~A}$ & 0.0348 & 0.0594 & 1.1762 & $0.105^{*}$ \\
\hline $\mathrm{C} 21$ & $0.54001(12)$ & $0.21462(11)$ & $0.76982(7)$ & 0.0564 (4) \\
\hline $\mathrm{C} 22$ & $0.55370(18)$ & $0.20232(16)$ & $0.71676(9)$ & $0.0865(7)$ \\
\hline $\mathrm{H} 22 \mathrm{~A}$ & 0.5097 & 0.1784 & 0.6959 & $0.104^{\star}$ \\
\hline $\mathrm{C} 23$ & $0.6331(3)$ & $0.2257(2)$ & 0.69471 (15) & $0.1281(14)$ \\
\hline $\mathrm{H} 23 \mathrm{~A}$ & 0.6420 & 0.2166 & 0.6590 & $0.154^{\star}$ \\
\hline $\mathrm{C} 24$ & $0.6976(2)$ & $0.2613(2)$ & $0.7238(2)$ & $0.1343(16)$ \\
\hline $\mathrm{H} 24 \mathrm{~A}$ & 0.7501 & 0.2778 & 0.7083 & $0.161 *$ \\
\hline
\end{tabular}

Table 2: (continued)

\begin{tabular}{lrrrr}
\hline Atom & $\boldsymbol{x}$ & $\boldsymbol{y}$ & $\boldsymbol{z}$ & $\boldsymbol{U}_{\text {iso }}{ }^{*} \boldsymbol{U}_{\text {eq }}$ \\
\hline C25 & $0.68524(16)$ & $0.27284(19)$ & $0.77621(17)$ & $0.1163(12)$ \\
H25A & 0.7299 & 0.2964 & 0.7967 & $0.140^{*}$ \\
C26 & $0.60637(13)$ & $0.24967(15)$ & $0.79948(10)$ & $0.0787(6)$ \\
H26A & 0.5986 & 0.2580 & 0.8353 & $0.094^{*}$ \\
C27 & $0.63538(11)$ & $0.03058(11)$ & $0.86225(7)$ & $0.0525(4)$ \\
C28 & $0.65541(13)$ & $0.08140(13)$ & $0.90562(7)$ & $0.0608(5)$ \\
H28A & 0.6151 & 0.0849 & 0.9328 & $0.073^{*}$ \\
C29 & $0.73312(14)$ & $0.12755(14)$ & $0.91056(7)$ & $0.0678(5)$ \\
H29A & 0.7444 & 0.1615 & 0.9405 & $0.081^{*}$ \\
C30 & $0.79341(12)$ & $0.12296(14)$ & $0.87107(8)$ & $0.0668(5)$ \\
C31 & $0.77609(13)$ & $0.07094(15)$ & $0.82747(8)$ & $0.0696(5)$ \\
H31A & 0.8172 & 0.0663 & 0.8008 & $0.084^{*}$ \\
C32 & $0.69846(12)$ & $0.02608(14)$ & $0.82328(7)$ & $0.0631(5)$ \\
H32A & 0.6876 & -0.0084 & 0.7935 & $0.076^{*}$ \\
C33 & $0.8949(2)$ & $0.2210(3)$ & $0.91319(16)$ & $0.1465(14)$ \\
H33A & 0.9522 & 0.2453 & 0.9079 & $0.220^{*}$ \\
H33B & 0.8940 & 0.1861 & 0.9449 & $0.220^{*}$ \\
H33C & 0.8537 & 0.2695 & 0.9159 & $0.220^{*}$ \\
H1 & $0.0248(12)$ & $0.3816(12)$ & $0.9669(6)$ & $0.056(5)^{\star}$ \\
H4 & $0.5056(16)$ & $0.1731(16)$ & $0.9129(9)$ & $0.092(8)^{\star}$ \\
H2 & $0.0570(12)$ & $0.2609(13)$ & $1.0066(8)$ & $0.065(6)^{\star}$ \\
\hline & & & &
\end{tabular}

\section{Experimental details}

The $\mathrm{H}$ atoms were calculated geometrically and refined as riding, with $\mathrm{C}-\mathrm{H}=0.93-0.98 \AA$ with $U_{\text {iso }}(\mathrm{H})=1.2 U_{\text {eq }}$ (parent atom).

\section{Comment}

$N$-(5-(2-(Benzyl(1-(4-methoxyphenyl)propan-2-yl)amino)1-hydroxyethyl)-2-(benzyloxy)phenyl)formamide is an important precursor to prepare Formoterol, which is a widely used pharmaceutical active compound [5-7]. Herein, we report the crystal structure of the title compound. In the title compound, all the bond lengths are within normal ranges [8-10]. The molecules of the title compound are interlinked to from 3D supramolecular networks by two classic intermolecular $\mathrm{H}$-bondings O4-H4-N1 (length 2.735(2) $\AA$, angle 120.18(4) ${ }^{\circ}$ ) and N2$\mathrm{H} 2-\mathrm{O} 4(x-1 / 2,-y+1 / 2,-z+2)$ (length 2.910(3) A, angle $\left.144.42(19)^{\circ}\right)$.

Acknowledgements: We are very grateful to Shandong Province Inorganic and Analytical Chemistry Excellent Resource Sharing Course (2018) and Luan Hui-ni Famous Teacher Studio (2019). 
Author contributions: All the authors have accepted responsibility for the entire content of this submitted manuscript and approved submission.

Research funding: None declared.

Conflict of interest statement: The authors declare no conflicts of interest regarding this article.

\section{References}

1. BRUKER. SAINT, APEX2 and SADABS; Bruker AXS Inc.: Madison, Wisconsin, USA, 2009.

2. Sheldrick G. M. A short history of SHELX. Acta Crystallogr. 2008, A64, 112-122.

3. Brandenburg K. DIAMOND. Visual Crystal Structure Information System, Version 2.0f; Crystal Impact: Bonn, Germany, 1998.

4. Yu X., Xiao D., Zheng G., Chen Y., Yuan X. Preparation Method of Formoterolas $\beta 2$ Sympathomimetic Agent, its Pharmaceutically Acceptable Salt and Intermediate; Assignee Guangzhou Joincare Respiratory Drugs Engineering Technology Co., Ltd.; Shanghai Front Health Pharmaceutical Technology Co., Ltd. CN 109535027A, 2019.
5. Al-Salama Z. T., Frampton J. E. Glycopyrronium/formoterol: a review in COPD. Drugs 2019, 79, 1455-1466.

6. O’Byrne P. M., FitzGerald J. M., Bateman E. D., Barnes P. J., Zhong N., Keen C., Jorup C., Lamarca R., Ivanov S., Reddel H. K. Inhaled combined budesonide-formoterol as needed in mild asthma. N. Engl. J. Med. 2018, 378, 1865-1876.

7. Tang B., Wang J., Luo L. L., Li Q. G., Huang D. Risks of budesonide/ formoterol for the treatment of stable COPD: a meta-analysis. Int. J. Chronic Obstr. Pulm. Dis. 2019, 14, 757-766.

8. Lu Z.-S., Zhu G.-Z., Shi H.-L., Wang X.-S. Crystal structure of $\beta$-[[(1,1-dimethylethoxyl)-carbonyl]amino]benzenepropanoic acid, $\mathrm{C}_{14} \mathrm{H}_{19} \mathrm{NO}_{4}$. Z. Kristallogr. NCS 2014, 229, 215-216.

9. Yang F.-L., Chen X., Wu W.-H., Zhang J.-H., Zhao X.-M., Shi Y.-H., Shen F. Spin switching in tris(8-aminoquinoline)iron(II) $\left(\mathrm{BPh}_{4}\right)_{2}$ : quantitative guest-losing dependent spin crossover properties and single-crystal-to-single-crystal transformation. Dalton Trans. 2019, 48, 231-241.

10. Yang F.-L., Liang B.-B., Zhang J.-H., Wu W.-H., Zheng S.-Q., Mao Y.-Y., Chen J.-Q., Cao J.-X., Zhu G.-Z. Synthesis, structures and magnetic properties of three copper(II) complexes of 2-(1H-pyrazol-3-yl) pyridine. Transit. Met. Chem. 2018, 43, 211-220. 